\title{
Influence of severe plastic deformation in phase transformation of superduplex stainless steels
}

\section{ArticleCategory : Metals}

Copyright-Holder: Springer Science+Business Media, LLC, part of Springer Nature

Copyright-Year: 2018 Núria Llorca-Isern ${ }^{1}$, Alisiya Biserova-Tahchieva ${ }^{1^{*}}$, Isabel Lopez-Jimenez ${ }^{1}$, Irene Calliari ${ }^{2}$, Jose Maria Cabrera
${ }^{3}$, Antoni Roca ${ }^{1}$

1 Department of Materials Science and Physical Chemistry, University of Barcelona, Martí i Franquès 110, 08028, Barcelona, Spain

2Department of Industrial Engineering, University of Padova, Via Gradenigo, 6/a, 35131, PadovaPadua, Padova, Italy

${ }^{3}$ Department of Materials Science and Metallurgical Engineering, Polytechnic University of Catalonia, Eduard Maristany 10-14, 08019, Barcelona, Spain

Rec: 20 June 2018, Accp: 4 October 2018,

\section{Abstract}

Duplex and superduplex stainless steels are characterised by high corrosion resistance and high mechanical strength. However, these steels can suffer formation of secondary brittle phases when they reach temperatures between $600^{\circ} \mathrm{C}-600$ and $950^{\circ} \mathrm{C} 950^{\circ} \mathrm{C}$, which can lead to the catastrophic service failure of components. In order to understand the influence of the mechanical history of the steel part, equal-channel angular pressing was applied followed by different thermal treatments. Microstructural characterisation was carried out on the ECAPed samples before and after thermal treatment. The analysis of the hardness evolution of the same samples was also evaluated.

\section{Introduction}

The microstructure of duplex stainless steels is balanced as $50 \%$ austenite $(\gamma)$ and $50 \%$ ferrite $(\delta)$ which leads to the steels with an excellent combination of mechanical and chemical properties. Crucially, duplex stainless steels possess high strength and corrosion resistance. However, for a particular range of temperatures $600^{\circ} \mathrm{C}$ to $1000^{\circ} \mathrm{C} 600-1000^{\circ} \mathrm{C}$, secondary intermetallic phases, such as sigma phase $(\sigma-$ phase), chi phase ( $\mathrm{X}$-phase) and chromium nitrides and carbides, precipitate. This promotes a matrix impoverishment in critical alloying elements, i.e. chromium, molybdenum and nickel, that leads to decrease in toughness values and corrosion resistance in these high-alloyed steels [1- $\mathbf{6}]$. The $\sigma$-phase increases the hardness and decreases the toughness as well as the elongation of this type of steels [ $\mathbf{7}$ ] and can change the fracture type from transgranular to intergranular, which is related to an increase in the $\sigma$-phase percentage [8]. The $X$-phase precipitates as a ternary compound containing $\mathrm{Fe}, \mathrm{Cr}$ and $\mathrm{Mo}[\underline{\mathbf{2}}, \mathbf{9}]$ with a wide range of stoichiometry extending from the ternary $x$-phase $\mathrm{Fe}_{36} \mathrm{Cr}_{12} \mathrm{Mo}_{10}$ to $\mathrm{Fe}_{36} \mathrm{Cr}_{12} \mathrm{Mo}_{3} \mathrm{Ti}_{7}$ depending on the steel composition $[\mathbf{1 0}]$. The $X$-phase and $\sigma$-phase can usually be detected.

The effect of equal-channel angular pressing (ECAP) on the precipitation of these secondary phases in duplex and superduplex stainless steel (UNS S32205 and UNS S32750) has not yet been studied. Many studies have reported this processing mode in pure or low-alloyed metals [11, 12], but very few have studied this process in stainless steels [13], and none of them involve ECAP and phase transformation. Therefore, the objective of this research is to study the microstructural changes,-as well as the evolution of mechanical properties in superduplex stainless steel (SDSS) after ECAP and compare this to the unprocessed samples [2]. 


\section{Materials and methods}

The materials used in this study were superduplex stainless steel grade 2507 (UNS S32750, in this study SDSS 2507). The chemical composition is shown in Table $\underline{\underline{\mathbf{1}}}$.

Table 1 Chemical composition of SDSS 2507 (\%wtwt\%, balance Fe)

\begin{tabular}{|l|l|l|l|l|l|l|l|l|l|}
\hline $\mathrm{C}$ & $\mathrm{Si}$ & $\mathrm{Mn}$ & $\mathrm{P}$ & $\mathrm{S}$ & $\mathrm{Cr}$ & $\mathrm{Ni}$ & $\mathrm{Mo}$ & $\mathrm{N}$ & $\mathrm{Cu}$ \\
\hline 0.018 & 0.260 & 0.840 & 0.019 & 0.001 & 25.080 & 6.880 & 3.820 & 0.294 & 0.170 \\
\hline
\end{tabular}

Cylinder samples of length $38.3 \mathrm{~mm} 3 \mathrm{~mm}$ length and diameter $5 \mathrm{~mm}-5 \mathrm{~mm}$ were machined from a tube with an internal diameter of $168.3 \mathrm{~mm} 3 \mathrm{~mm}$ and a wall thickness of $11 \mathrm{~mm}-11 \mathrm{~mm}$ in order to expose them to the ECAP die (with an ECAP angle of $120^{\circ}$ and in warm condition of processing, $230^{\circ} \mathrm{C} 230^{\circ} \mathrm{C}$ ). The tone-pass ECAP specimen was firstly cut longitudinally and subsequently one of the halves was cut transversally in order to apply different thermal treatments (Figure-Fig. $\underline{\mathbf{1}}$ ).

\section{3_2018_2984_Fig1_print.png}

Fig. 1 Figure 1 Zones of sample extraction for microstructural characterisation and heat treatments. The extrusion direction (ED), normal direction (ND) and transverse direction (TD) are indicated. Nomenclature for half cross - section is also shown

The thermal treatment was carried out on the half-samples after a procedure of aging ageing at $830 \pm 2{ }^{\circ} \mathrm{C}$ $830 \pm 2{ }^{\circ} \mathrm{C}$ from three minutes 3 to ten minutes 10 min followed by water quenching. Metallographic conventional sample preparation was achieved by grinding and polishing with diamond pastes. The microstructural analysis was conducted using scanning electron microscope FESEM JEOL J-7100F with a coupled Robinson BSE detector. The microhardness was performed with Galileo ISOSCAN OD microhardness tester applying $0.2 \mathrm{Kg}$-f during $15 \mathrm{~s} 15 \mathrm{~s}$.

\section{Results and discussion}

Transverse and longitudinal analysis of samples was performed by means of high-resolution microscopy (FESEM) in order to analyse possible microstructural transformations which can occur after processing the SDSS 2507 samples (Figure-Fig. 2).

\section{3_2018_2984_Fig2_print.png}

Fig. 2 Figure 2 Schematic representations of the mechanism followed by the austenite during ECAP showing the modification of its shape and distribution

\section{Transverse section}

The transverse sections before and after one-pass ECAP are shown in Figure-Fig. $\underline{\mathbf{3}}$. Although microstructure does not show abrupt changes, the morphology of ferrite and austenite presents a more acute phase boundary if compared to the unprocessed sample (Figure-Fig. $\underline{\mathbf{3}} \mathrm{a}, \mathrm{b}$ ) in which flat interphases boundaries are observed. Furthermore, it is noted that, for transverse sections, slightly differences of the width or size are observed, which are related to the deformation process (Figure-Fig. $\underline{\mathbf{3}} \mathrm{c}, \mathrm{d}$ ).

\section{3_2018_2984_Fig3_print.png}

Fig. 3 The microstructure-Figure 3 Microstructure of SDSS transversal section, bright phase austenite phase-austenite $(\mathrm{Y})$ and darker grey phase ferrite-phase-ferrite $(\delta)$. Before one-pass ECAP (a)-and (b) and after one-pass ECAP (c) and (d)

After one-pass ECAP, however, in some areas the austenite phase becomes finer and forms grouped islands, as shown in Figure-Fig. 4. This particular feature is not observed in the unprocessed sample and is found throughout over the cross section and therefore cannot be attributed to a particular location near or far from the inner angle of the ECAP device. These islands are most likely the consequence of certain rotational movements of the phases, mostly austenite leading to a sort of phase fractioning and separation. 
with circles)

\section{Longitudinal section}

The longitudinal section before and after one-pass ECAP is shown in Figure-Fig. $\underline{\mathbf{5}}$. Contrary to what is observed in the transverse section, the longitudinal microstructure changes significantly. As shown, the austenite is thinner than in the unprocessed ECAP sample. Moreover, some bridging of austenite phase at angles ranging from $37^{\circ}$ to $45^{\circ}$ connects austenite bands (Figure-Fig. $\underline{\mathbf{5}} \mathrm{d}$ ). These austenite bands are probably the unusual morphology observed in the transverse sections, described as islands in Figure-Fig. 4 , and can be attributed to shear forces from the process creating these austenite shear bands.

\section{3_2018_2984_Fig5_print.png}

Fig. 5 The microstructure-Figure 5 Microstructure of SDSS longitudinal section before one-pass ECAP (a) and after one-pass $\operatorname{ECAP}(\mathbf{b}, c, d, e,-\mathbf{f})$

Isolated islands of austenite can also be observed in Fig. $\underline{\mathbf{5}} \mathrm{c}$. Fig. Figure $\underline{\mathbf{5}} \mathrm{b}$ corresponds to the upper part of the specimen affected by the inner ECAP radius, whereas Fig. $\underline{\mathbf{5}}$ is the bottom part affected by the outer ECAP angle. In Fig. $\underline{\mathbf{5}}$, extensive polygonisation has been observed, whereas --more bridging has occurred in Fig-Fig. $\underline{5}$ b.

After the SDSS specimen was passed through ECAP, the corresponding microstructure indicated strong changes in the austenite. Some of the mechanisms modifying the shape and distribution of the austenite in duplex stainless steels can be attributed to plastic deformation, whereas others are the result of strain partitioning, strain localisation and some phase accommodation [14]. Plane strain or simple shear deformation are observed when the uniform plastic deformation of both ferrite and austenite is produced. On the other hand, shear banding, rotation, displacement by sliding on the interphase, fragmentation by shear banding, bulging, fragmentation by penetration of ferrite into austenite along GB and polygonalisation are frequently observed observed in the austenite in SDSS. These mechanisms are typically found during hot working forming processes.

SEM characterisation of the ECAPed samples provides evidence of phase rotation that promotes the change of the ferrite-austenite-ferrite-austenite interphase boundaries. Hence, it is assumed that the initial orientation relationship (K-S or N-W) also experiences a lattice rotation, which modifies the interphase boundary coherency, especially if subgrains are developed in the ferrite. In addition to the presence of lattice rotations on the substructural scale, macroscopic phase rotations are also observed. Austenite can rotate as a whole within the ferrite matrix, which has simultaneously experienced intense local shearing.

As shown in Figure-Fig. $\underline{\mathbf{6}}$, due to shear strain, voids in the austenite phase are formed (and filled by ferrite penetration) resulting in longitudinal fragmentations of austenite.

\section{3_2018_2984_Fig6_print.png}

Fig. 6 The microstructure-Figure 6 Microstructure of SDSS longitudinal section after one-pass ECAP: a) Polygonisation polygonisation, austenite rotation, b)-large irregularities and $\mathbf{c}$ )-small irregularities in the interphase boundaries are clearly shown

After ECAP the interphase boundary remains flat in some areas. However, throughout the sample, the interphases become mobile and some perturbations take place on a large scale leading to some interpenetration of both ferrite in austenite and austenite in ferrite. Interphase boundary sliding involving the translation of a grain with respect to another by a shear movement parallel to their common boundary , -has also been observed. Sliding affects the interphase but does not happen at ferrite-ferrite or austenite-austenite boundaries. Sliding depends on the character of the boundary. It is known that coherent and semi-coherent interfaces are less prone to sliding than incoherent boundaries. When deformation is applied to as-cast and wrought specimen, sliding at the interphase between the two phases, ferrite and austenite, in K-S relationship in duplex stainless steel does not take place, whereas -in hot-rolled steel, sliding occurs justified by the loss of the K-S relationship [14]. This can be interpreted as the retention of K-S orientation relationship in deformed as-cast and cold work specimen, but its loss in hot forming processes. In the present work, sliding has been found in the SDSS specimen after one-pass ECAP (no K-S orientation relationship kept) as well as large resultant damage of interphase boundary decohesion or microcracks nucleated at the austenite block tip (K-S orientation relationship kept during SPD), as shown in Figure-Fig. $\mathbf{7}$. Therefore, after ECAP the K-S 
orientation relationship between austenite and ferrite is mostly retained.

\section{3_2018_2984_Fig7_print.png}

Fig. 7 The microstructure-Figure 7 Microstructure of SDSS longitudinal section after one-pass ECAP, with decohesion at the austenite-ferrite interphase boundaries marked on the image

SDSS have-has a high tendency for strain localisation and shear band formation. The presence of two phases with significantly different mechanical strengths can be considered as the primary cause for shear band formation.

Significant microstructural fragmentation has been observed in combination with deformation at low deformation temperatures. For duplex stainless steels at hot workability conditions, it has been previously shown that, for temperatures less than approximately $1000^{\circ} \mathrm{C} 1000^{\circ} \mathrm{C}$, shear bands crossing the interphase boundary can produce some fragmentation of the austenite by shearing [14]. Furthermore, for deformation temperatures above $1000^{\circ} \mathrm{C} 1000^{\circ} \mathrm{C}$, interphase mobility is high enough to produce some bulging and some local scale rearrangements in the microstructure, according to the following sequence: interphase boundary bulging, formation of triple points at the intersection between the interphase boundary and both austeniteaustenite boundaries and ferrite-ferrite grain boundaries of segmented-type ferrite. Bulging of the austenite into ferrite and some penetration of ferrite into austenite single-phase boundary leads at the limit to fragmentation of austenite stringers into individual crystallites. ECAPpedECAPed duplex stainless steel showed significant fragmentation of austenite. However, the mechanism does not appear to be caused by the formation of shear bands crossing the interphase boundary or by interphase boundary bulging out of austenite into ferrite accompanied by some penetration of ferrite into austenite single-phase boundary. Rather, this mechanism can be attributed to large coherent boundary sliding in austenite followed by the formation of stringers of austenite initially bridging the longitudinal fragments perpendicularly. As the localised shearing deformation increases, the stringers are strained which leads to tearing in the shearing direction and deflecting of towards the shearing angle. Some tearing can be large enough to cause ruptures. If the shearing is too large or the stringer is too thin, an irregularity of the austenite in the austenite-ferrite boundary forms, rather than a developed stringer (Figure-Fig. $\underline{\mathbf{5}}$ ). During this process, the rearrangement of ferrite in the microstructure takes place.

As discussed, sliding on the interphase has been identified as a source of damage due to its coherency. As shown in Figure-Fig. $\underline{\mathbf{6}}$, decohesion at the coherent interphase boundary between an austenite ridge and ferrite can clearly be observed. Boundary sliding contributes to strain accommodation in the SDSS during hot working. Strain partitioning, for example, requires some amount of sliding on the interphase boundaries in order to maintain continuity. Strain localisation and disperse phase rotations also induce some sliding. For example, the austenite block rotation is accompanied by severe shearing in the surrounding soft ferrite and by damage formation at several points on the interphase boundary. In superduplex microstructures shear stresses also appear as a result of the presence of two phases with different mechanical properties. Furthermore, under certain conditions, SDSS are-is softer when deformed under pure shear mode than under plain strain conditions which enhances the formation of shear bands.

\section{Microhardness tests}

The microhardness of the samples was measured before and after one-pass ECAP. The average hardness of the sample before the ECAP, in the cylinder shape, was $263 \pm 4263 \pm 4 \mathrm{HV}$. Hardness evolution through the sample, from north to south -and,-west to east, is shown in Figure-Fig. $\underline{\mathbf{8}}$. The average hardness for the sample after one-pass ECAP is $332 \pm 10-332 \pm 10 \mathrm{HV}$. As expected, after one - pass the average hardness of the SDSS increased in the samples used for this study. This value is lower than expected after one-pass ECAP. The increase in hardness produced in other bulk materials after SPD treatments tends to double with respect to unprocessed samples. For the superduplex stainless steel, this can be attributed to the fragmentation of grains occurring during ECAP [13].

\section{3_2018_2984_Fig8_print.png}

Fig. 8-Figure 8 Hardness of perpendicular distribution after one-pass ECAP ( $N$ to $S$ in blue and W to $E$ in red indicated at the schema) in the transverse section

The north region is affected by the inner radius of ECAP, and the south region is affected by the outer radius. As shown, functional work induces hardening $(325 \mathrm{HV})$, thereby; therefore areas show a decrease in 
microhardness (minimum values of $306 \mathrm{HV}$ ), reaching the highest values at the upper central region of the sample (338-364-338-364 HV). From west to east, the eenterline-centreline of hardness measurements is indicated in the schema shown in Figure-Fig. $\mathbf{8}$. It showed higher values with the coincident one at the eenter centre of the cross-sectionsectional sample.

Neither longitudinal nor transverse sections contain secondary $\mathrm{X}$-phases and $\sigma$-phases. It can therefore be concluded that this precipitation does not occur after one-pass ECAP in a $120^{\circ}$ warm die. Annealing was carried at $830^{\circ} \mathrm{C} 830^{\circ} \mathrm{C}$ for different dwell periods in order to compare the influence of the severe plastic deformation with sufficient time necessary to precipitate $x$-phases or $\sigma$-phases, at their temperature range.

Peak stress has been found to rise with increasing $\mathrm{Cr}$ and Mo content, and the relative effect of Ni was found to be negligible. Previous studies have shown that $\mathrm{N}$ content induces a planar dislocation structure detrimental to the cell or subgrains substructure [14]. Some studies investigating the maximum stress as a function of $\mathrm{Cr}$ eq. to $\mathrm{Ni}$ eq. ratio found the highest stress in SDSS compositions, followed by DSS [14]. Furthermore, within each group, the stress decreases systematically with an increasing $\mathrm{Cr}$ eq. to $\mathrm{Ni}$ eq. ratio [14]. Mo exhibits the highest partitioning to ferrite, whereas $\mathrm{C}$ and $\mathrm{N}$ exhibit the lowest. In this sense, chromium nitrides are observed only after the ECAP process and as they are closely related with the formation of secondary phases in duplex stainless steels, their location has been studied. They are found mainly at the austenite-ferrite interphase boundaries and, more precisely, at the newly formed interphase boundaries of the austenite stringers.

\section{Thermal Freatmentstreatments}

The corresponding micrographs are shown in Figure-Fig. 9. All the thermal treatments were performed on the half-samples of one-pass ECAP. From the microstructural analysis, it is found that secondary precipitation has occurred. As shown in Figure-Fig. $\underline{\mathbf{9}}$, the microstructure at different holding times indicates an increase in the amount of secondary phases. After 10 -minutes-min treatment, the $\sigma$-phase almost completely dominates the ferrite. Nitrides and carbides are detected in the precipitation regions as dark spots.

\section{3_2018_2984_Fig9_print.png}

Fig. 9 The microstructure-Figure 9 Microstructure of SDSS after $830^{\circ} \mathrm{C}-830^{\circ} \mathrm{C}$ thermal treatment for: a) and b) 3 minutes $3 \mathrm{~min}, \mathbf{c}$ ) and d) 5 minutes $5 \mathrm{~min}$ and, e) and f) 10 minutes $10 \mathrm{~min}$, transversal and longitudinal section, respectively

In the longitudinal section of the samples, the precipitation of $\sigma$-phases and $x$-phases after 3 minutes 3 min increases from north to south. Furthermore, it was found for some of these samples, $\sigma$-phases and $x$-phases nucleated following a longitudinal pattern among ferrite and austenite interphase boundaries (Figure-Fig. 10). It is worth noticing that in the case of samples aged for 5 and 10 minutes 10 min the quantity of $X$-phases phase is lower than that of the 3 minutes 3 min thermally treated sample; however, however the $\sigma$-phase in longer aging ageing time is more extended all over the entire sample.

\section{3_2018_2984_Fig10_print.png}

Fig. 10 The microstructure-Figure 10 Microstructure of SDSS after one-pass ECAP followed by 3 minutes aging 3 min ageing, showing the elongated morphology of $\sigma$-phases and $x$-phases

In the transverse sections, it is observed that $\sigma$-phase precipitation ECAP in the upper region increases significantly from north to south. This can be attributed to regions of high HV. Very few signs of $\sigma$-phases and $x$-phases are found in the upper region of the sample which was annealed for 3 minutes 3 min. Even fewer are found in the lower-most region (ECAP outer angle region). Conversely, in the 5 -minutes agingmin ageing sample, the lower-most exhibits more $\sigma$-phase than the north zone of the same sample. The sample which was aged for 10 minutes 10 min has a more homogeneous distribution amount of $\sigma$-phase. Furthermore, the extreme west area of the centre line along the TD direction exhibits higher content of secondary phases (Figure-Fig. 11).

\section{3_2018_2984_Fig11_print.png}

Fig. 11 The microstructure-Figure 11 Microstructure of SDSS after one-pass ECAP followed by 3 minutes aging3 min ageing: western region of the centre line of TD

From the presented results it is found that, compared to unprocessed samples, the time required to 
precipitate secondary phases at the same temperature is lowered when the sample is previously ECAP processed using a $120^{\circ}$ warm die. A similar behaviour has been verified in a cold-rolled 2205 [15]. The precipitation temperature and the amount of dislocations generated during the SPD process are not correlated. The results confirm that the precipitation mechanism - and hence the formation of the secondary brittle phases, is are enhanced. The partition of some elements involved in this precipitation seems to be favoured too, and even reinforced. Finally, the rotation and the movement of interphase boundaries as well as the penetration of both delta and gamma phases in each other favours the diffusional processes involved in the partitioning rearrangement of alloying elements.

\section{Conclusions}

The microstructure of superduplex stainless steel (UNS S32750 grade) produced by one-pass ECAP at $120^{\circ}$ warm die is similar to that produced in hot rolling of this alloy. Chromium nitrides are clearly observed after the ECAP process. However, neither $\sigma$-phases and-nor $x$-phases were detected after the SPD process. The precipitation of the secondary $\sigma$-phases and $X$-phases occurred after the ECAP-processed samples were thermally treated. This precipitation nucleated at austenite-ferrite boundaries, following a longitudinal pattern among ferrite and austenite interphase boundaries. The boundaries between ferrite and austenite stringers formed during shear deformation are preferential sites for secondary precipitation nucleation. This precipitation occurred within a shorter dwell time at the same ageing temperature than for non-ECAP-processed specimens.

The increase in hardness produced by the ECAP processing is significant and not found homogeneously all over the sample. The highest value in hardness was found in the centre part of the specimen.

\section{Compliance with ethical standards}

\section{Conflict of interest}

The authors declare that they have no conflict of interest.

\section{References}

1. Nilsson JO, Wilson A (1993) Influence of isothermal phase transformations on toughness and pitting corrosion of super duplex stainless steel SAF 2507. J -Mater -Sci -Technol -9:545-554

2- Llorca-Isern N, López-Luque H, López-Jiménez I, Biezma MV (2016) Identification of sigma and chi phases in duplex stainless steels. Mater -Charact $-112: 20-29$

3. S. Bernhardsson,-Bernhardsson S (1991) The corrosion resistance of duplex stainless steels-. Duplex Stainless Steels'91, vol 1(1991), pp 185-210=

4. Chen T-Chen, Weng K.Weng, J. Yang, Yang J (2002) The effect of high-temperature exposure on the microstructural stability and toughness property in a 2205 duplex stainless steel,. Mater -Sci -Eng -, A .338 (2002) 259-270.338:259-270

5- Sieurim H. Sieurim, R. Sandström, Sandström R (2007) Sigma phase precipitation in duplex stainless steel 2205-. Mater -Sci -Eng -,A -444(2007) 271-276.444:271-276

6-: Padilha AF, Tavares CF, Martorano MA (2012) Delta Ferrite Formation in Austenitic Stainless-Steel Gastingsferrite formation in austenitic stainless-steel castings. Mater -Sci -Forum -730-732:733-738

7. 1. K. JooSuk LEE, Application of Small Punch Test to Evaluate Sigma Phase Embrittlement of Pressure VesselCladding Material, J. Nucl. Sci. Technol. 40 (2003) $664-671$.
8. Lee JS, Kim IS (2003) Application of small punch test to evaluate sigma-phase embrittlement of pressure vessel cladding material. J Nucl Sci Technol 40:664-671

8 Biezma MV, Berlanga C, Argandona G (2013) Relationship between microstructure and fracture types in a UNS S32205 duplex stainless steel. Mater -Res -16:965-969

9. Y.H.Lee YH, K.T.Kim KT, Y.D. Lee K. Y. Kim, Lee YD, Kim KY (1998) Effects of W substitution on $\varsigma$ and X phase precipitation and toughness in duplex stainless steels-. Mater -Sci -Technol -14(1998) 757 
10- Gunn R (1997) Duplex stainless steels: microstructure, properties and applications. Elsevier, New York

11. Valiev RZ, Langdon TG (2006) Principles of equal-channel angular pressing as a processing tool for grain refinement. Prog-Mater-Sci -51:881-981

12- t.J.Beyerlein IJ, R.A. Lebensohn RA, G.N. Tomé, PolycrystalConstitutive Modeling Tomé CN (2002) Polycrystal constitutive modeling of ECAP: Fexture and Microstructural Evolution, in: Ultrafine Grained Mater. II, John Wiley \& Sons, Ine., 2002.13:texture and microstructural evolution. In: Ultrafine grained mater. II. Wiley

13 Ajith PM, Sathiya P, Gudimetla K, Ravishankar B (2013) Mechanical, MetallurgicalCharacteristics and Gorrosion Properties of Equal Channel Angular Pressing of Duplex Stainless Steel. Adv. Mater. Res. metallurgical characteristics and corrosion properties of equal channel angular pressing of duplex stainless steel. Adv Mater Res 717:9-14

14. Ł.Alvarez-Armas I, S.Degallaix-Moreuil -S (2009) Duplex stainless steel, John. Wiley-\&sons, 2009.Hoboken 